\title{
ON THE NATURE OF THE VENUS CLOUDS
}

\author{
GERARD P. KUIPER \\ University of Arizona, Tucson, Ariz., U.S.A.
}

\begin{abstract}
A comparison of a wide range of Venus observations with the ultraviolet, visible, and infrared reflection spectra and thermodynamic properties of a variety of candidate materials indicates that the principal constituent of the Venus clouds is partially hydrated $\mathrm{FeCl}_{2}$.
\end{abstract}

\section{Introduction}

Hypotheses on the composition of the Venus clouds have proliferated in recent years. The Proceedings of the CalTech-JPL Lunar and Planetary Conference [1] describe the following interpretations, held by different authors: small water droplets, small ice crystals, hail stones (at depth); derivatives of methane, ethane, and benzene; and dust. Other recent publications have considered snow flakes (at depth), $\mathrm{NH}_{4} \mathrm{Cl}$, an ice- $\mathrm{HCl}$ solution, volcanic products, a carboniferous swamp, a planetary oil field, and the 'global-seltzer ocean' theory. A useful critical view of this curious assortment is made in the NASA Handbook of Venus [2]. Of necessity, many questions had to be left open for lack of data.

With the very low, but finite, mixing ratio of $\mathrm{H}_{2} \mathrm{O} / \mathrm{CO}_{2}$ now established from the NASA 990 Jet observations $[3,4]$ and with the reinterpretation of the $2 \mu$ region of the Johns Hopkins balloon spectrum of Venus [5], coupled with the new polarization data of Venus by Coffeen [6], the problem of water or ice clouds can be dealt with definitively. One of the arguments used in past years in support of the ice hypothesis, the low reflectivity of the planet between 3-4 $\mu$, is reinterpreted on the basis of new spectrophotometric data and found to lead to a very different conclusion.

\section{Clouds Visible in Ultraviolet}

Ross [7] in his extensive photographic coverage of Venus in 1927, using the 60-inch Mt. Wilson telescope, established that the planet shows a variable cloud pattern in UV light ( $3600 \AA \pm$ ); but that in yellow or red light no clear markings appear except possibly on rare occasions. Wright [8] had previously taken a few photographs at the Lick Observatory with the same general result. The cloud pattern may show changes even in a few hours. Recently the French observers (Boyer and Camichel [9]; Boyer [10]; Boyer and Guérin [11] noted that the cloud pattern at times moves across the disk in retrograde motion roughly $90^{\circ}$ per day, corresponding to a pseudorotation period of 4-5 days. No strict periodicity exists, however, either in this motion of the upper atmospheric layers nor in the details of the cloud forms. The observed motion could obviously either be real or a group velocity caused by atmospheric waves. The conclusions by Boyer $e t$ al. have been corroborated by observations at the McDonald and Catalina Observatories, a selection of which is published in Comm. LPL No. 102. 
Ross [7] found that the UV clouds on Venus become gradually invisible at wavelengths substantially longer than $4000 \AA$. Recent observations at the Catalina Observatory by J. Fountain and S. Larson have shown that the cloud contrast continues to increase shortward of $3600 \AA$, down to at least $3200 \AA$. Undoubtedly, this contrast is related to the steep decrease of the planetary albedo (Figure 1). It would be of distinct interest to observe the cloud contrast telescopically from above the terrestrial ozone layer, between 2000-3000 $\AA$.

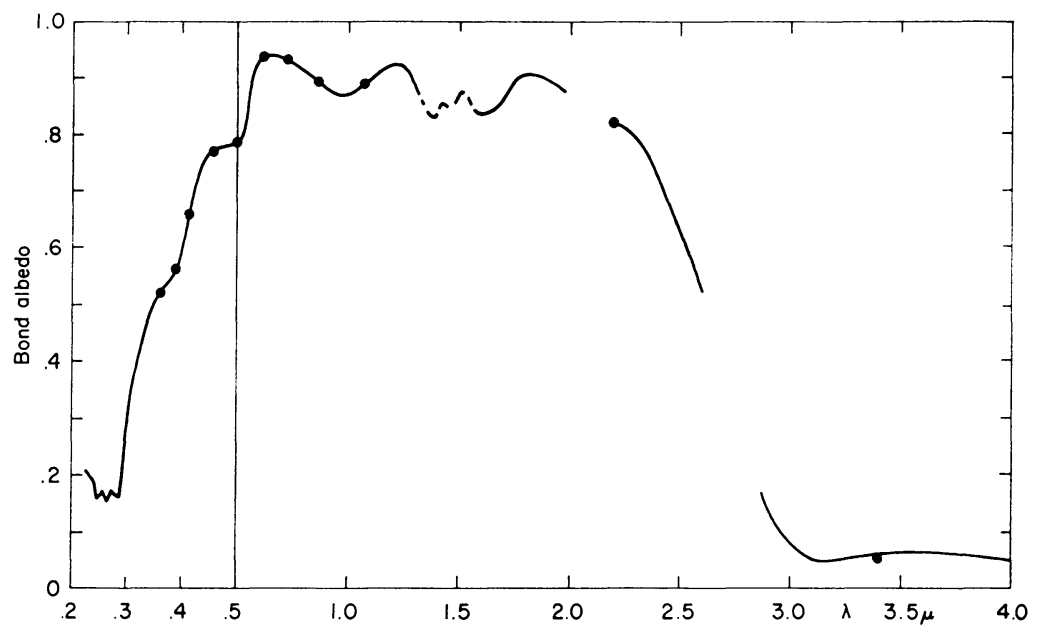

Fig. 1. Bond albedo vs. wavelength $0.2-4.0 \mu$. Details near $1.5 \mu$ somewhat uncertain.

\section{The Yellow Haze Layer}

Observations of the planet in yellow or red light normally show only indistinct markings. This is probably related to the excessively high albedo of the planet in yellow and red light (Figure 1) which must be attributed to a coefficient of scattering of the cloud particles very close to unity. As a result the cloud cover may be somewhat likened to a terrestrial snow surface without shadows. Yet it will be very important to ascertain whether breaks and irregularities may be seen with image resolution 10 or 100 times greater than achieved with earth-based telescopes.

The sources for Figure 1, which summarizes the data on the bond albedo of Venus for the wavelength interval $0.2-4.0 \mu$, have been described in a more extensive publication [12]. The curve has been corrected for absorptions by $\mathrm{CO}_{2}$, as derived from records with higher resolution. For the two gaps the $\mathrm{CO}_{2}$ absorptions were so large that allowance for them was not feasible. The region near $1.5 \mu$ is somewhat uncertain.

The albedo curve is clearly very informative on the nature of the Venus cloud cover. The broad absorptions have the appearance of being caused by a solid or a liquid. The identification of these absorptions has proceeded on the basis of laboratory studies. I am indebted to Rev. Godfrey Sill (O. Carm.) for major assistance in these laboratory comparisons and for helpful comments on the chemistry of the Venus atmosphere. This laboratory program is considered in Section 6. 


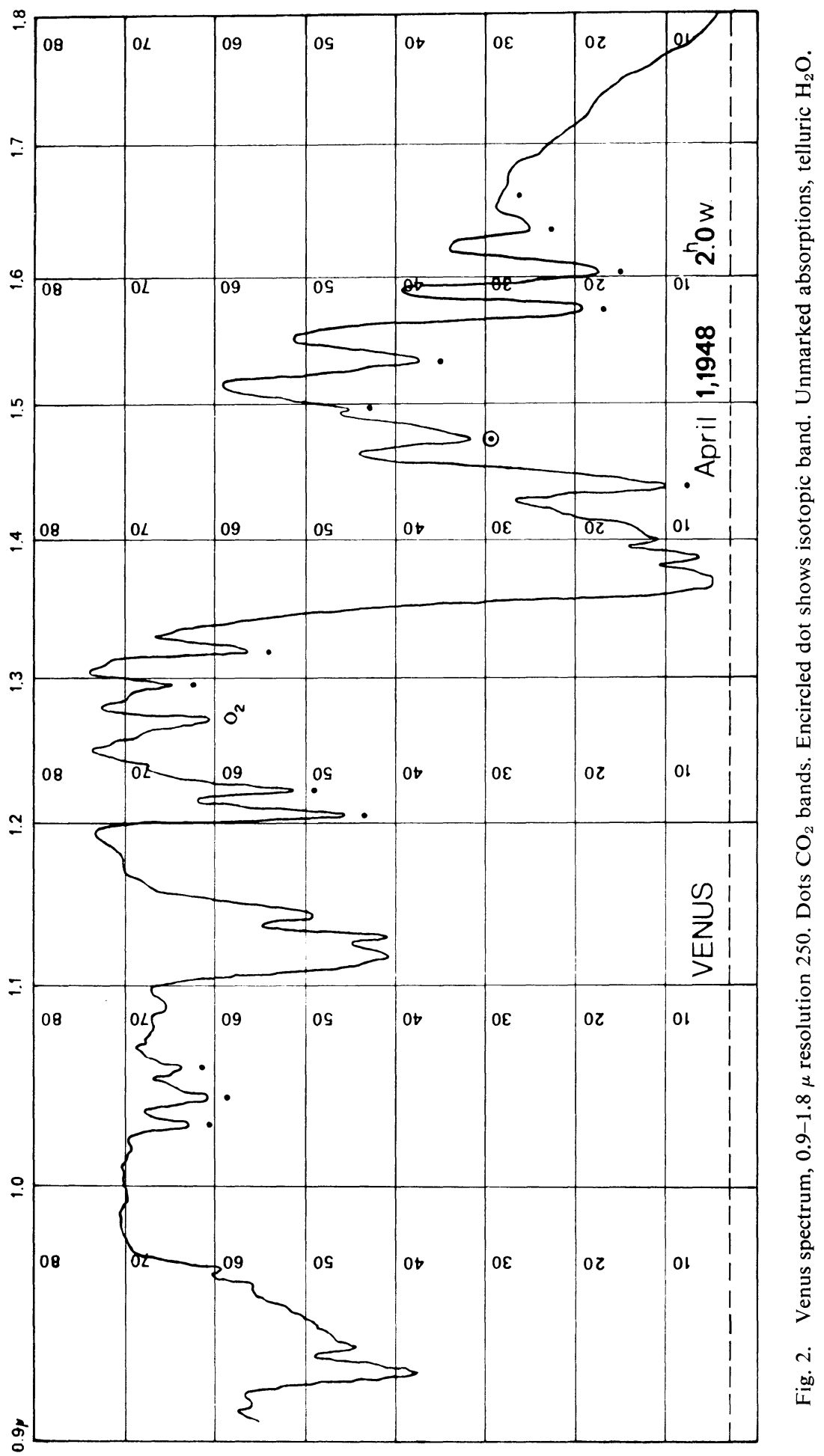




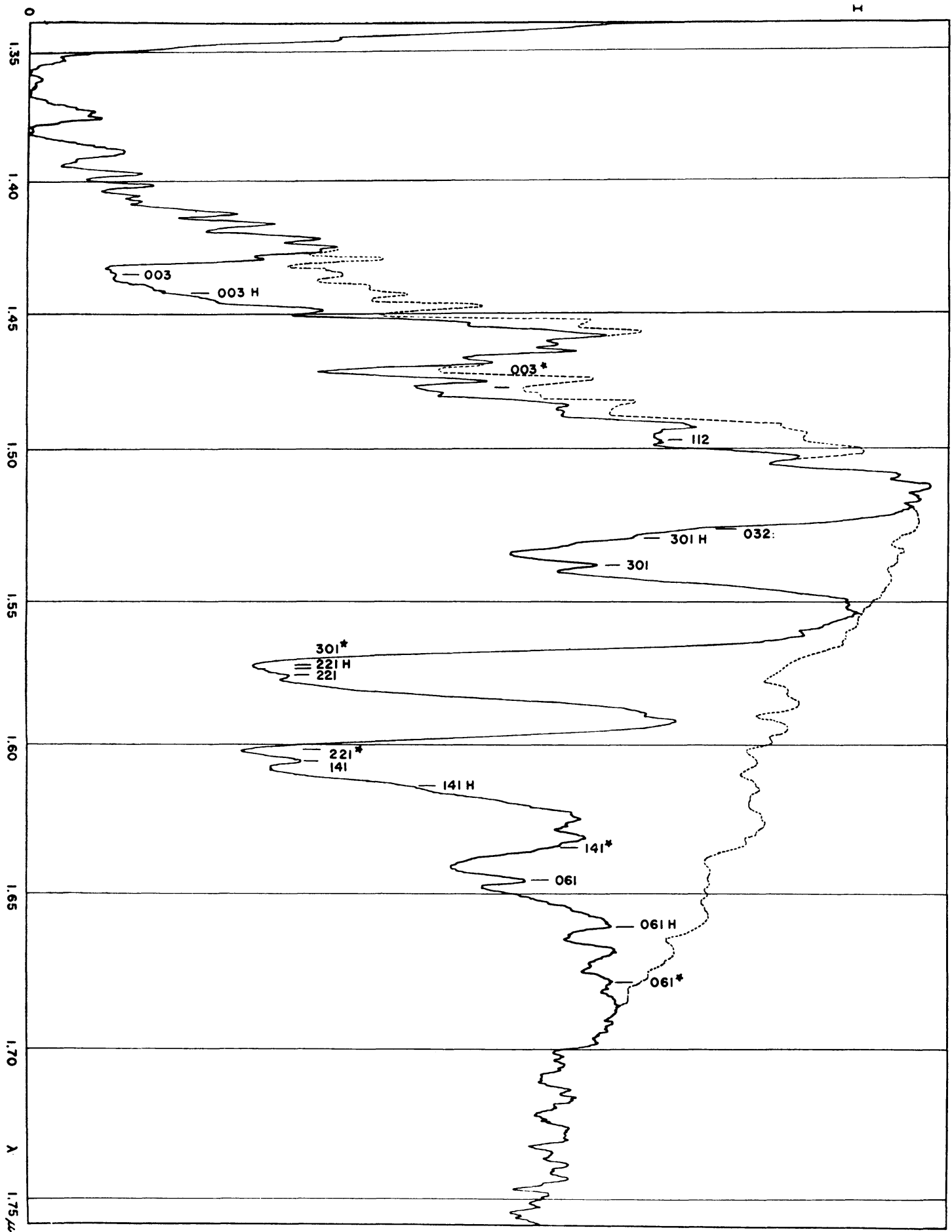

Fig. 3. Venus spectrum, KPNO 36", June 17, 1962, 1.35 $1.75 \mu$. Upper curve is solar spectrum. 


\section{Spectroscopy, Composition, and Temperature Profile of the Venus Atmosphere}

Substantial information has recently been derived on the atmosphere from spectroscopy in the near-infrared, $1-4 \mu$. These observations have all been made with the use of lead-sulfide cells, first developed during World War II. Reference is made to a spectrum obtained by the author with the 82-inch telescope of the McDonald Observatory, $0.9-1.8 \mu$, on April 1, 1948 (Figure 2), showing the now well-known strong $\mathrm{CO}_{2}$ bands, including the $\mathrm{C}^{13} \mathrm{O}_{2}$ isotopic band at $1.47 \mu$. This band was shown separated from the telluric $\mathrm{H}_{2} \mathrm{O}$ in spectra obtained in 1962 (Figure 3), allowing the first determination of $\mathrm{C}^{13} / \mathrm{C}^{12}$ for Venus (same as for the earth), and a similar determination of the $\mathrm{O}^{18} / \mathrm{O}^{16}$ ratio from the $2.15 \mu$ band.

During the last three years enormous progress in the IR spectroscopy of Venus and other planets has been achieved by the Connes [13] which led to the discovery of $\mathrm{HCl}$ and HF on Venus and set upper limits for a number of other gases. It also definitely established the presence of $\mathrm{CO}$ (made very probable previously by the work of Moroz [14]). The very-high resolution observations by the Connes, made at the Haute Provence (el. $650 \mathrm{~m}$ ), have been supplemented by the author with comparatively low-resolution interferometer observations from the NASA CV 990 at elevations $12-13 \mathrm{~km} \mathrm{[3,4]}$. These observations have definitely established the presence of water vapor in the Venus atmosphere [4]. They also showed the absence of ice absorptions near $2 \mu$ [3].

To illustrate the nature of the high-altitude interferometer spectra, we reproduce in Figure 4 the spectrum of the moon, used as comparison for the elimination of both telluric and solar absorptions, and one of the Venus spectra, Figure 5 (obtained Nov. 27-28, 1967). The ratio spectrum between the two will eliminate solar and remaining telluric absorptions and is given in Figure 6, together with identifications.

The utility of the high-altitude spectra may be judged by reference to corresponding ground-based spectra taken at the Catalina Observatory (el. $2580 \mathrm{~m}$ ). Figure 7 of the moon, Figure 8 the ratio spectrum Venus/Moon. They may be compared with Figures 4 and 6.

Table I collects the composition data on the Venus atmosphere presently available. A critical compilation of available data on the Venus temperature profile [4] is reproduced in Figure 9.

\section{Planetary Diameter and Cloud Level}

The published measures of the optical diameter of the planet are reviewed in the NASA Handbook [2]. The adopted mean value is that derived by de Vaucouleurs [15], $12240 \pm 15 \mathrm{~km}(R=6120 \pm 8 \mathrm{~km})$. This value coincides with the level of the tropopause in our Figure 9 and should be, within the uncertainties, equivalent to the upper boundary of the dense haze layer expected to form the visible limb of the planet. Subsequent photographic measures by the writer on the Venus crescent near inferior conjunction have given $R=6100$ for red and near-infrared light; and $R=6145 \mathrm{~km}$ 8 -P.A. 
GERARD P. KUIPER
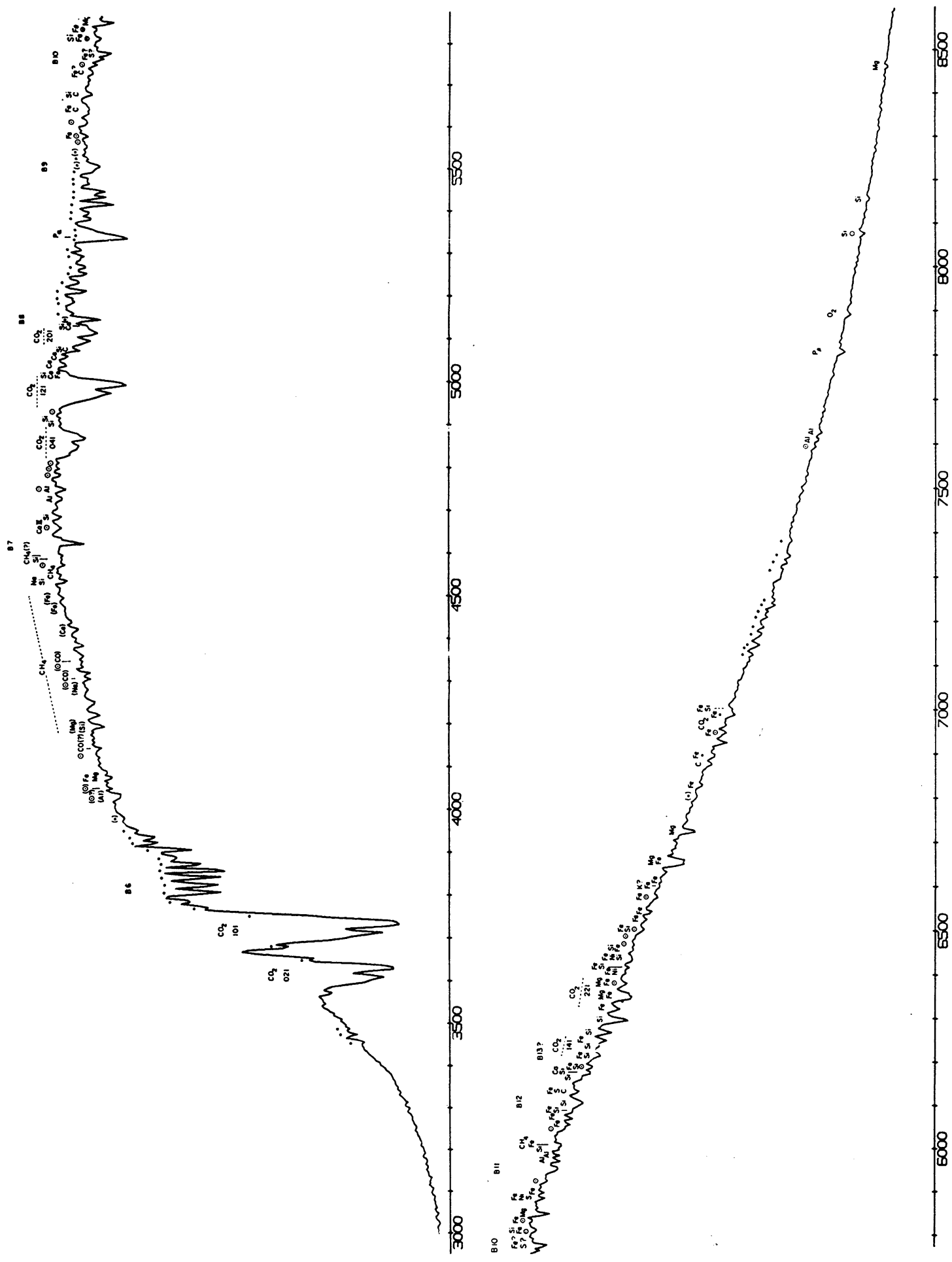

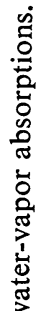

ชㅇㅇำ

己焉 

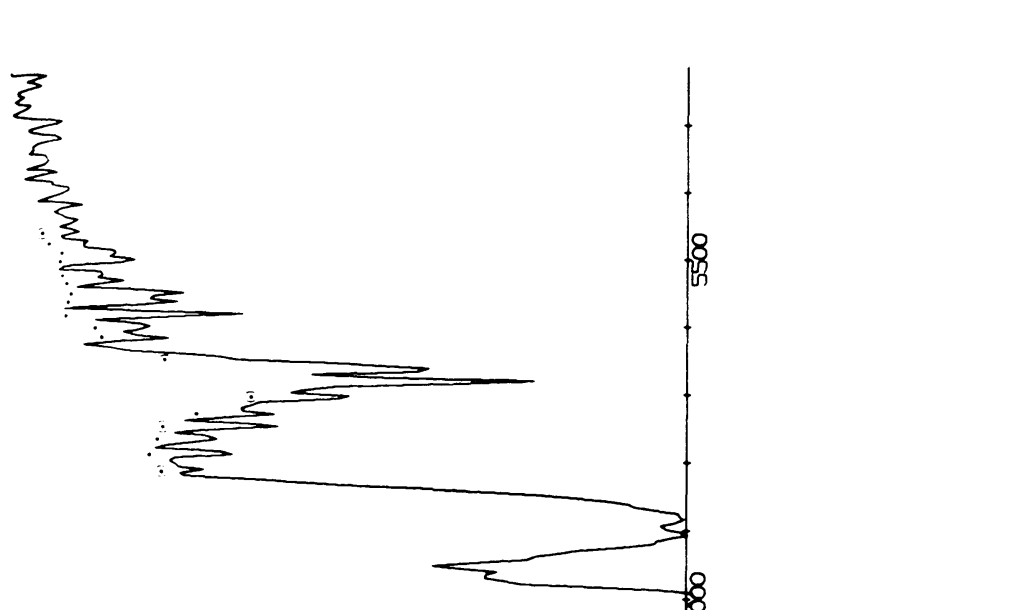
98
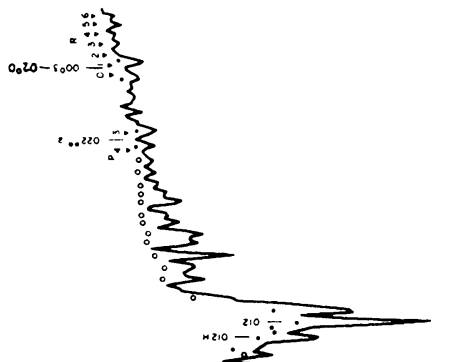

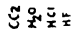
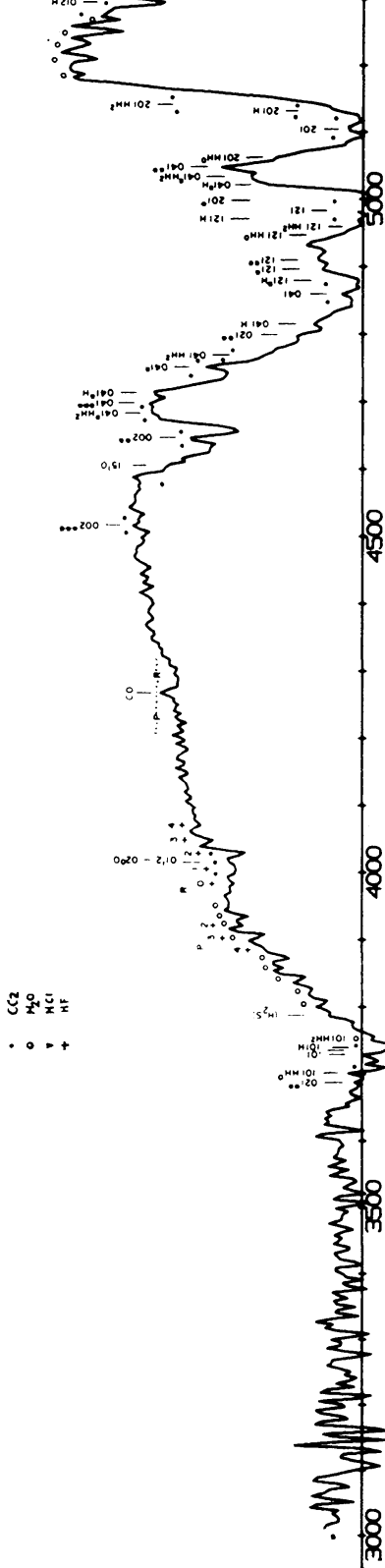

GERARD P. KUIPER
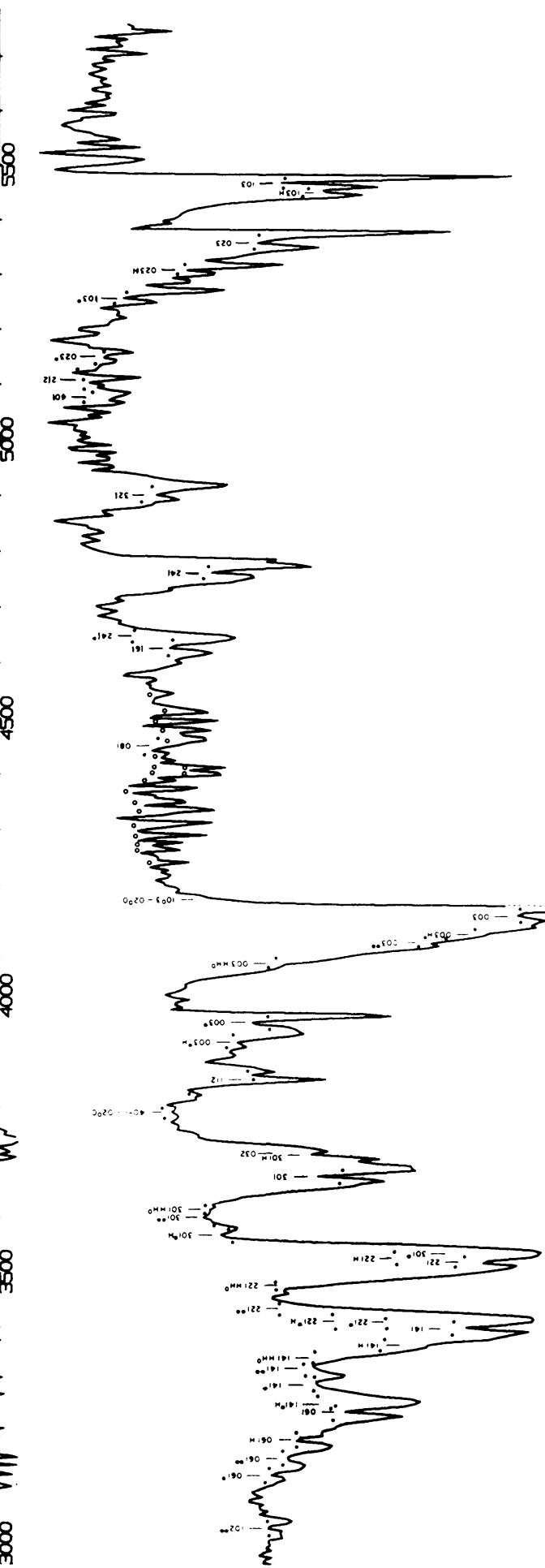

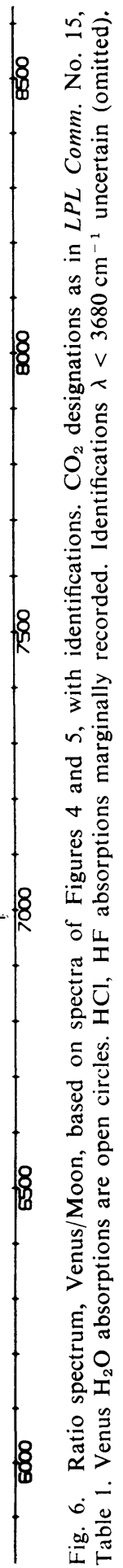



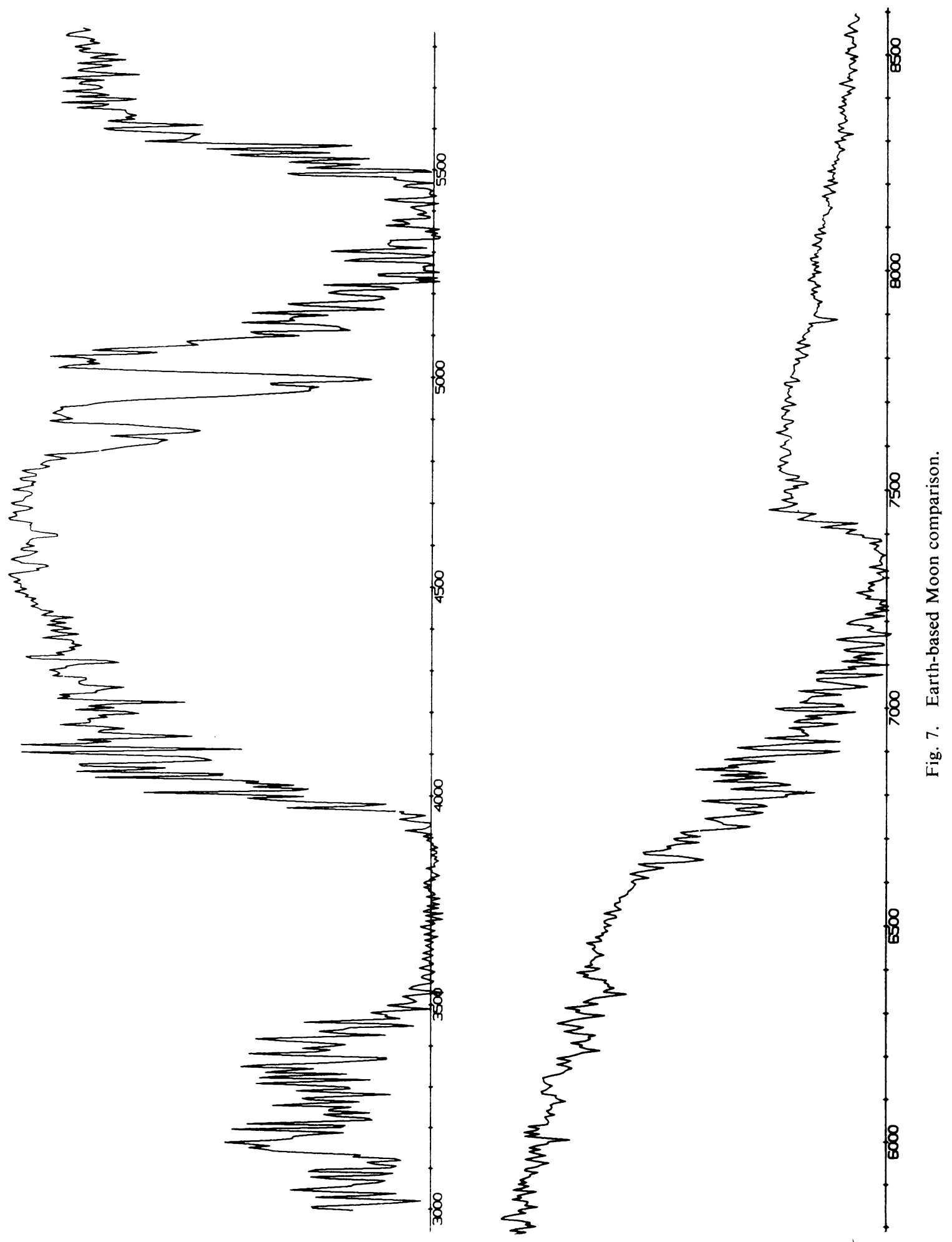
TABLE 1

Composition of Venus atmosphere

\begin{tabular}{lll}
\hline Gas & Mixing ratio & Source \\
\hline $\mathrm{CO}_{2}$ & $0.95 \pm 0.02$ & Venera 4, 5, 6 (Pravda, June 4, 1969). \\
$\mathrm{N}_{2}$ & $0.035 \pm 0.015$ & Venera 4, 5, 6 (Pravda, June 4, 1969). \\
$\mathrm{CO}$ & $10^{-4.34}$ & Connes et al., 1968, Astrophys. J. 152, 741. \\
$\mathrm{HCl}$ & $10^{-6.2}$ & Connes et al., 1967, Astrophys. J. 147, 1230-1237. \\
$\mathrm{HF}$ & $10^{-8.2}$ & Connes et al., 1967, Astrophys. J. 147, 1230-1237. \\
$\mathrm{CH}_{4}$ & $<10^{-6}$ & Connes et al., 1967, Astrophys. J. 147, 1235. \\
$\mathrm{CH}_{3} \mathrm{Cl}$ & $<10^{-6}$ & Connes et al., 1967, Astrophys. J. 147, 1235. \\
$\mathrm{CH}_{3} \mathrm{~F}$ & $<10^{-6}$ & Connes et al., 1967, Astrophys. J. 147, 1235. \\
$\mathrm{C}_{2} \mathrm{H}_{2}$ & $<10^{-6}$ & Connes et al., 1967, Astrophys. J. 147, 1235. \\
$\mathrm{HCN}$ & $<10^{-6}$ & Connes et al., 1967, Astrophys. J. 147, 1235. \\
$\mathrm{H}_{2} \mathrm{O}$ & $10^{-6.0}$ & Kuiper, G. P. et al., Comm. LPL No. 100. \\
$\mathrm{O}_{2}$ & $<10^{-5.0}$ & Belton and Hunten, 1968, Astrophys. J. 153, 970. \\
$\mathrm{O}_{3}$ & $<10^{-8}$ & Jenkins et al., 1969, Contrib. KPNO No. 421. \\
$\mathrm{SO}_{2}$ & $<10^{-7.5}$ & Cruikshank, D. P. and Kuiper, G. P.: Comm. LPL \\
& & No. 97. \\
$\mathrm{COS}$ & $<10^{-6}$ & Cruikshank, D. P., Comm. LPL No. 98. \\
$\mathrm{COS}$ & $<10^{-8}$ & Based on Comm. LPL No. 100. \\
$\mathrm{C}_{3} \mathrm{O}_{2}$ & $<10^{-6.3}$ & Based on Comm. LPL No. 100. \\
$\mathrm{H}_{2} \mathrm{~S}$ & $<10^{-3.7}$ & Cruikshank, D. P., Comm. LPL No. 98. \\
$\mathrm{NH}_{3}$ & $<10^{-7.5}$ & Based on Comm. LPL No. 100. \\
\hline
\end{tabular}

for the near UV ( $3600 \AA)$. This difference, of $0.7 \%$ is regarded real and appears to establish the reality of the separation of the UV haze layer from the top of the dense yellow haze, already inferred from its rapid horizontal motions.

\section{Identification of the Venus Haze Layer}

Figure 10 shows laboratory reflection curves of six substances powdered to about 50-100 $\mu$ particle size, investigated to determine whether silicate dust might be responsible for the yellow haze layer. The conclusion is clearly negative. (The slight positive features near 1.95 and $1.5 \mu$ are caused by minute water absorptions by the white standard composed of pulverized LiF. The rise shortward of $0.3 \mu$ in the silica curve and some others is likewise attributed to an imperfection of the white standard. Otherwise all reflection curves, including those of Figure 11, are accurate and in any case comparable.)

In Figure 11 sulfur and a selected number of carbonates and metallic halides are shown. These curves show enormous differences and only one substance, partiallyhydrated $\mathrm{FeCl}_{2}$, corresponds closely with the Venus reflection curve of Figure 1 . This material has an absorption at $0.24 \mu$ due to a charge transfer between $\mathrm{OH}$ and $\mathrm{Fe}$; at $0.29 \mu$ an absorption due to a charge transfer between $\mathrm{Cl}$ and $\mathrm{Fe}$; absorptions due to $d-d$ transfers of $\mathrm{Fe}^{2+}$ at $0.35,0.5,1.0$, and around $1.5 \mu$; and a deep absorption due to the hydrate centered on $3.0 \mu$. Each of these 7 absorptions is present, approximately in the expected amount, on Venus (Figure 1), and the identification is regarded certain. The only questions remaining pertain to the particle size (about $2.5 \mu$ for Venus and 50-100 $\mu$ for the laboratory samples) which affects the depth. not the warelength of 


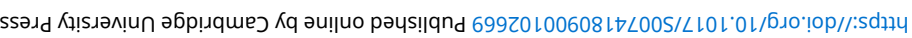

ON THE NATURE OF THE VENUS CLOUDS

101

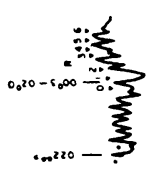
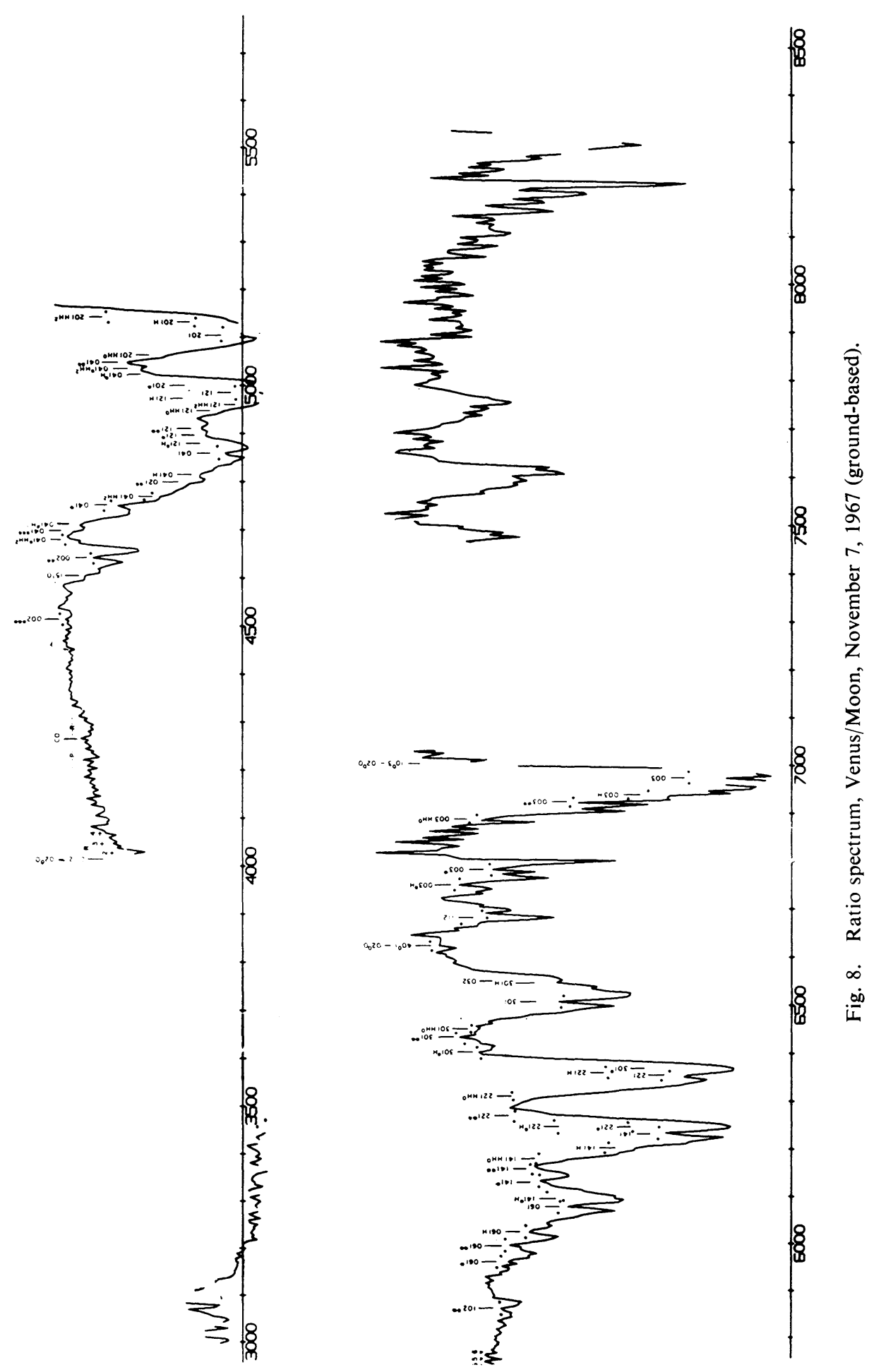
absorptions; and to the rise at $3.5-4.0 \mu$ in Figure $11 \mathrm{c}(q)$, not present in Venus. It is possible that this difference is due to the small particles of the Venus cloud layer (smaller than the wavelength) or else due to an admixture of a sulfate or a hydrate with an extended absorption beyond $3 \mu$ (cf. Figure 12). The refractive index of $\mathrm{FeCl}_{2} \cdot 2 \mathrm{H}_{2} \mathrm{O}$

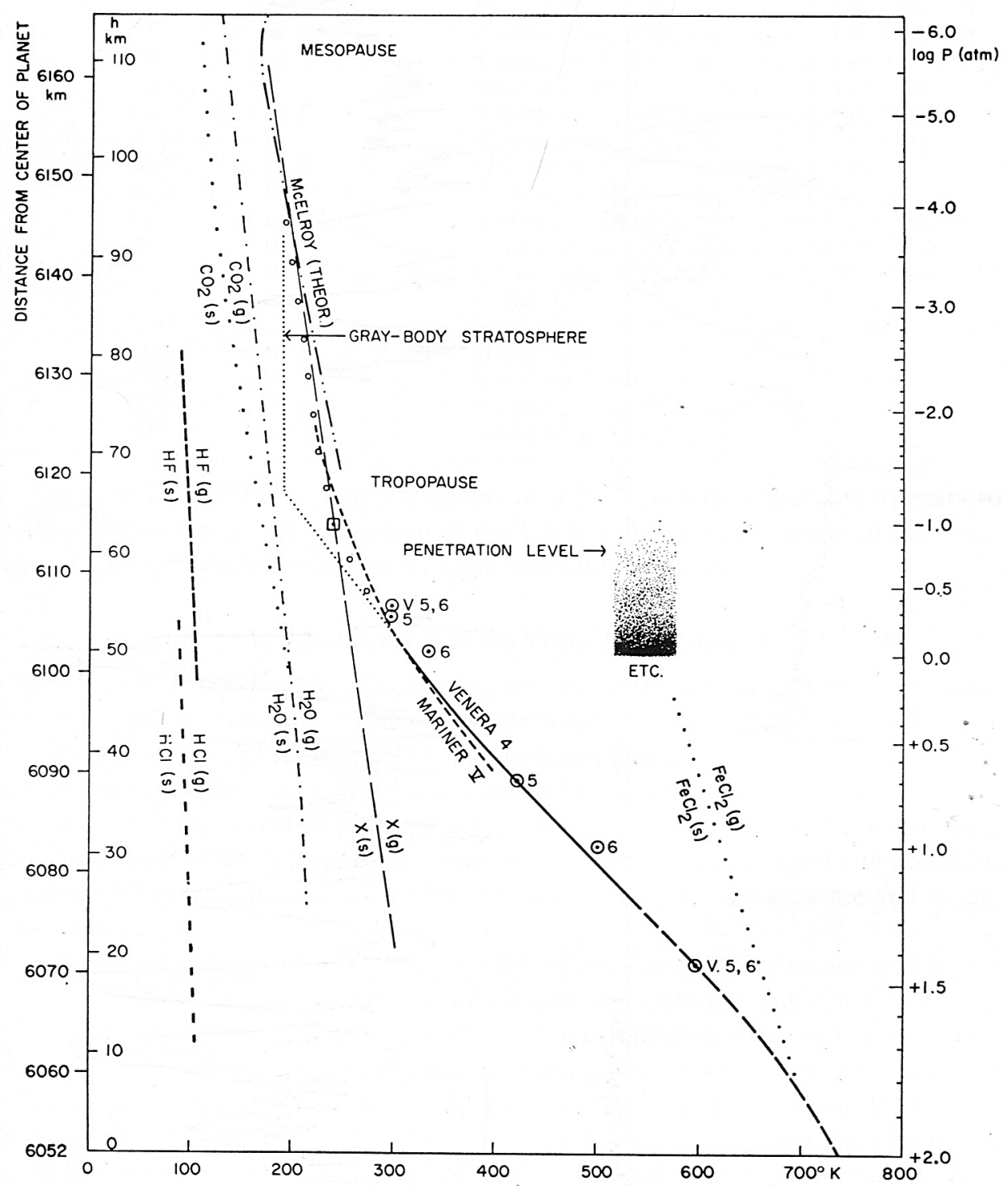

Fig. 9. Compilation of atmospheric pressure, temperature, and altitude relationships for planet Venus; dot in square, rotational temperature and pressure derived from CO (Connes et al., 1968); small open circles: relationship adopted in computation of pressures. 

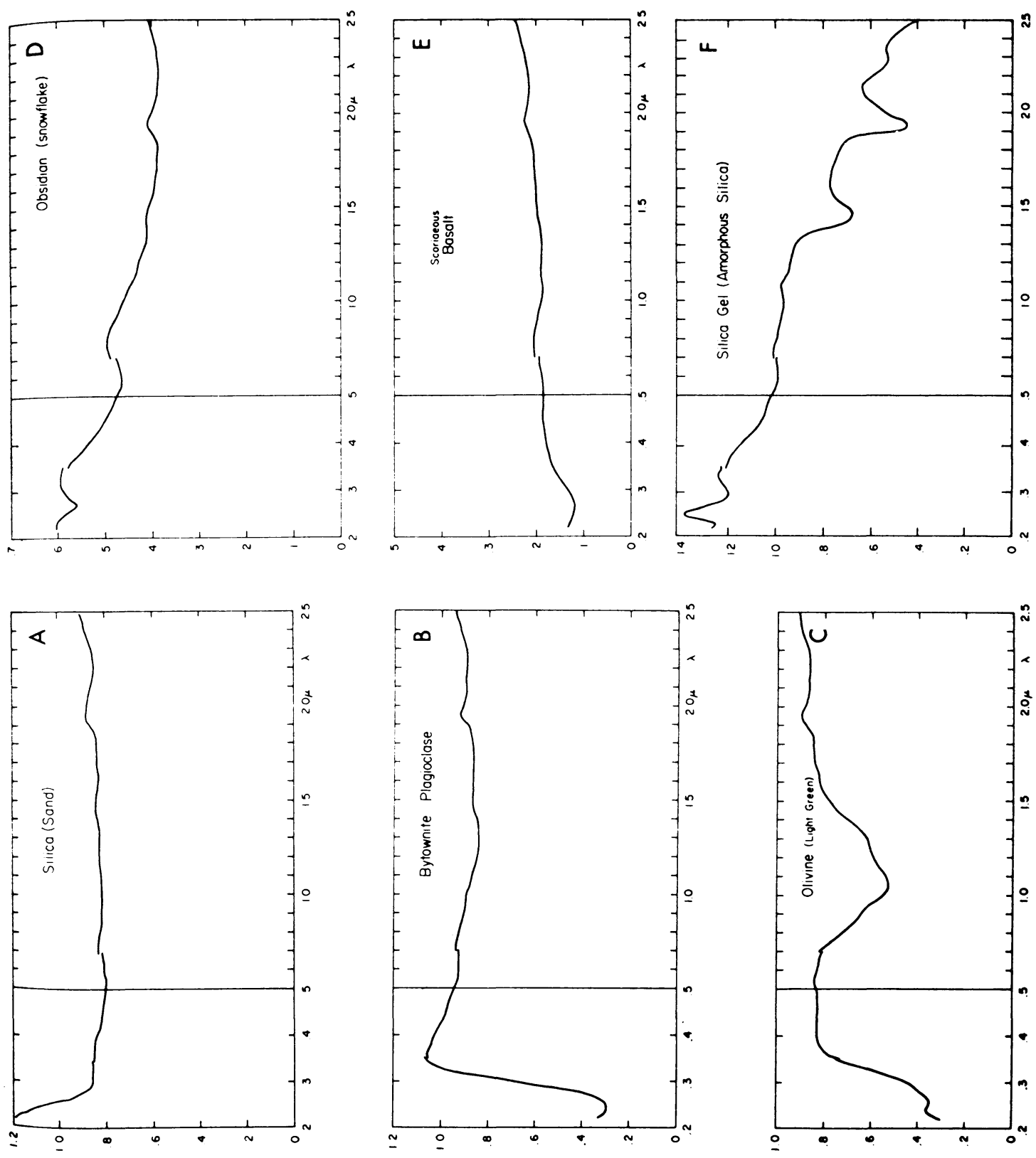

Fig. 10. Reflectance measurements of possible solid constituents in Venus atmosphere made by G. Sill (O. Carm.) with a Zeiss Reflectance Spectrophotometer, using a Li F (white) standard. 

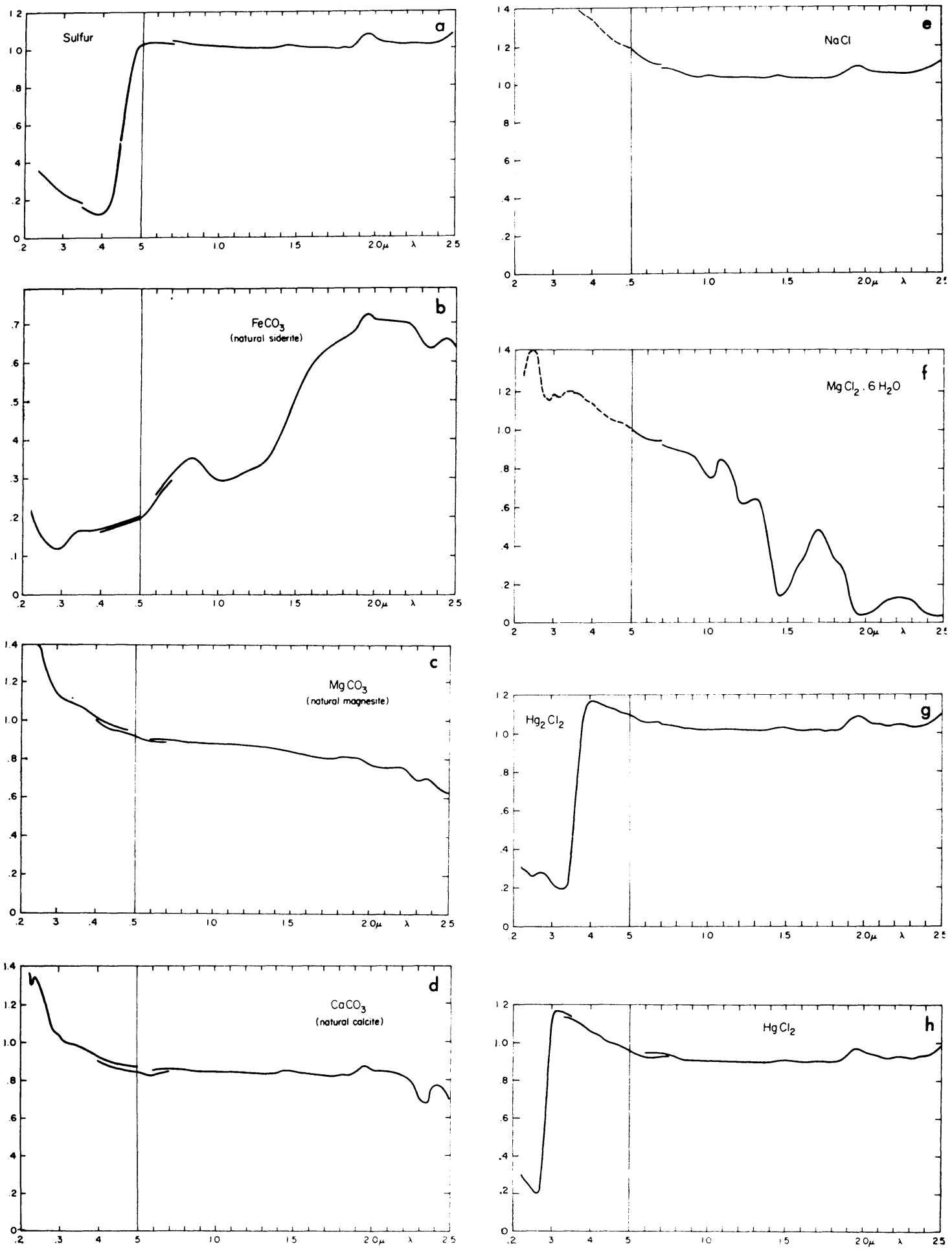

Fig. $11 \mathrm{a}$. 
ON THE NATURE OF THE VENUS CLOUDS
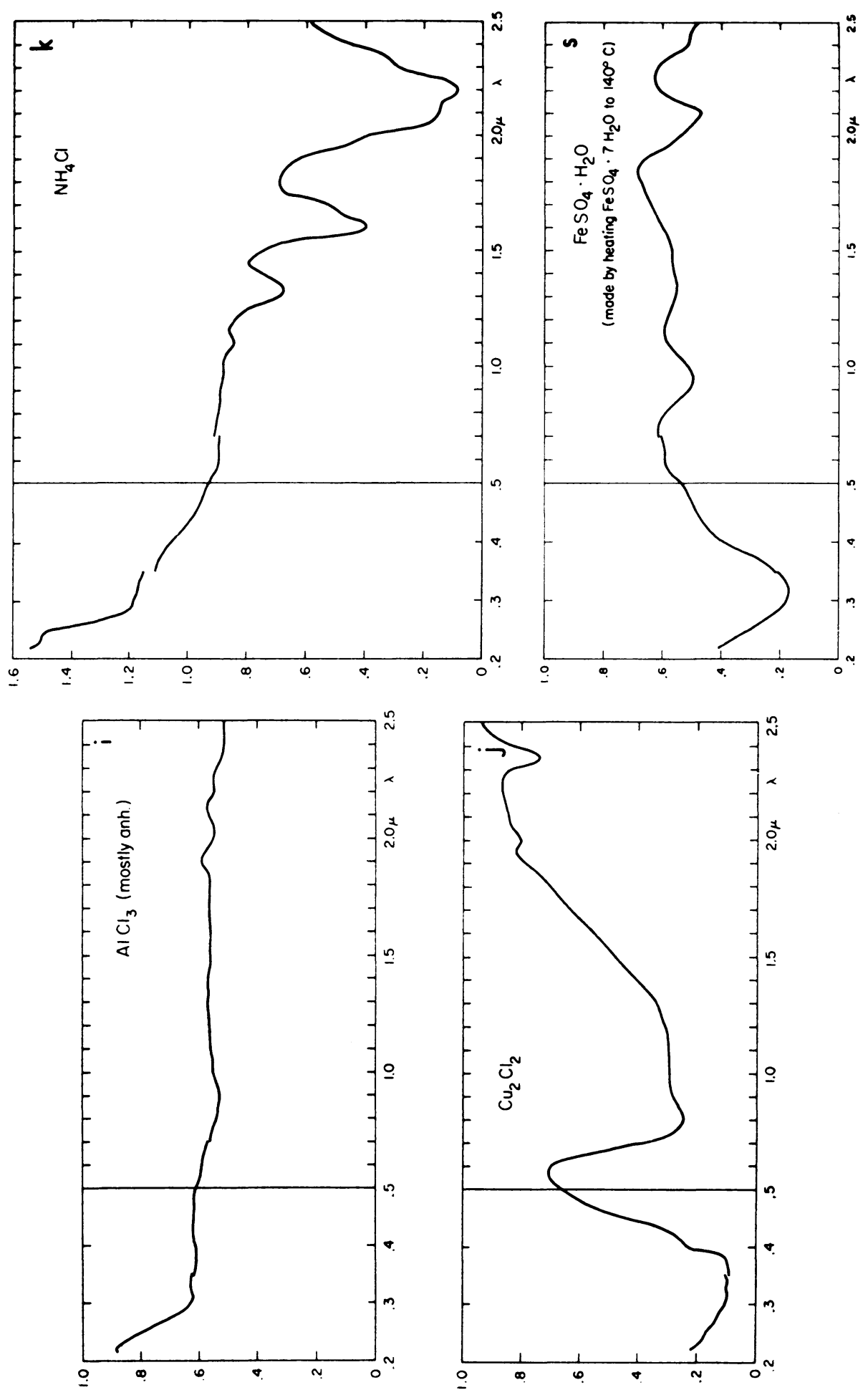

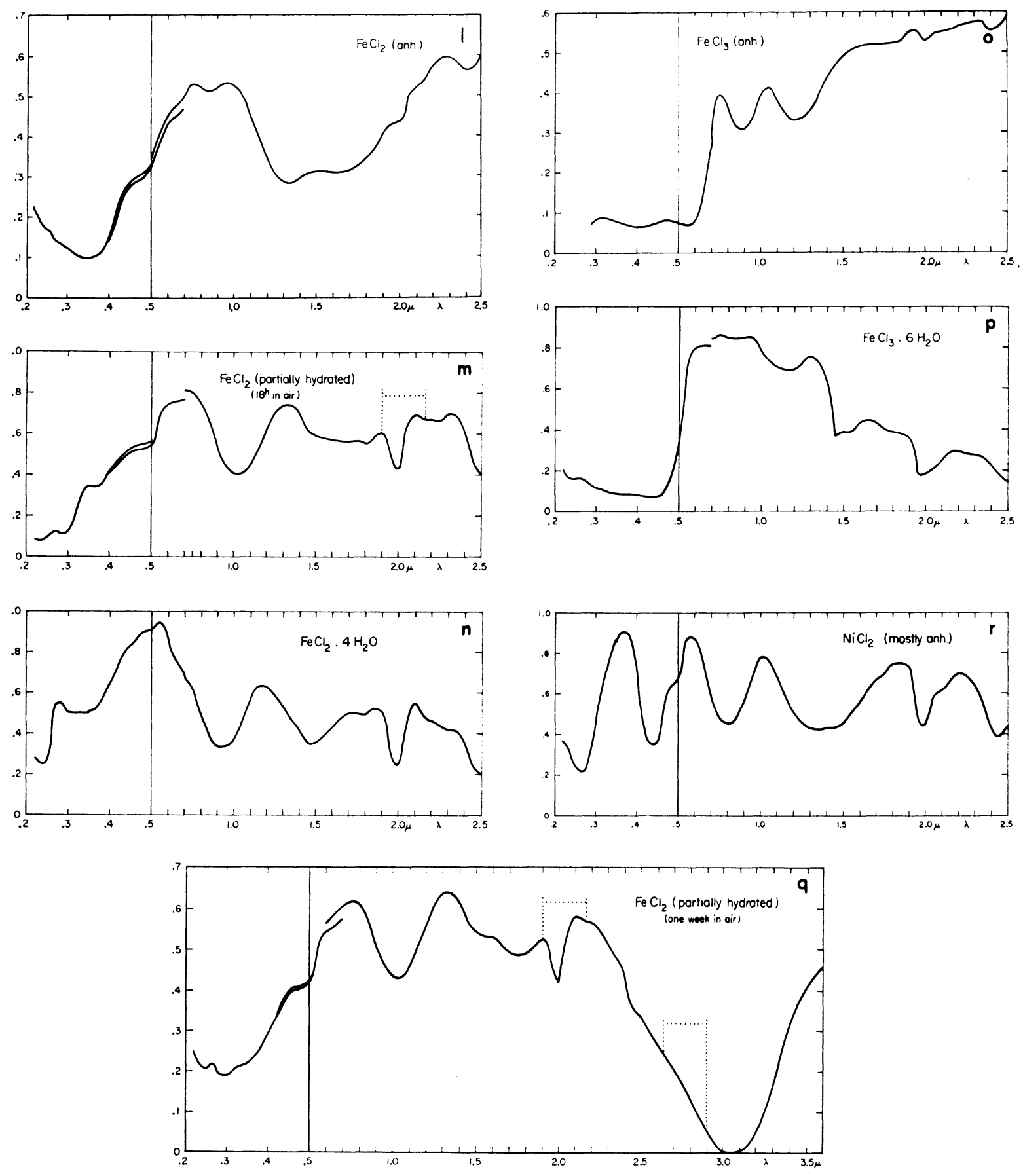

Fig. 11c. Reflectance curves of sulfur, carbonates and metallic halides. 

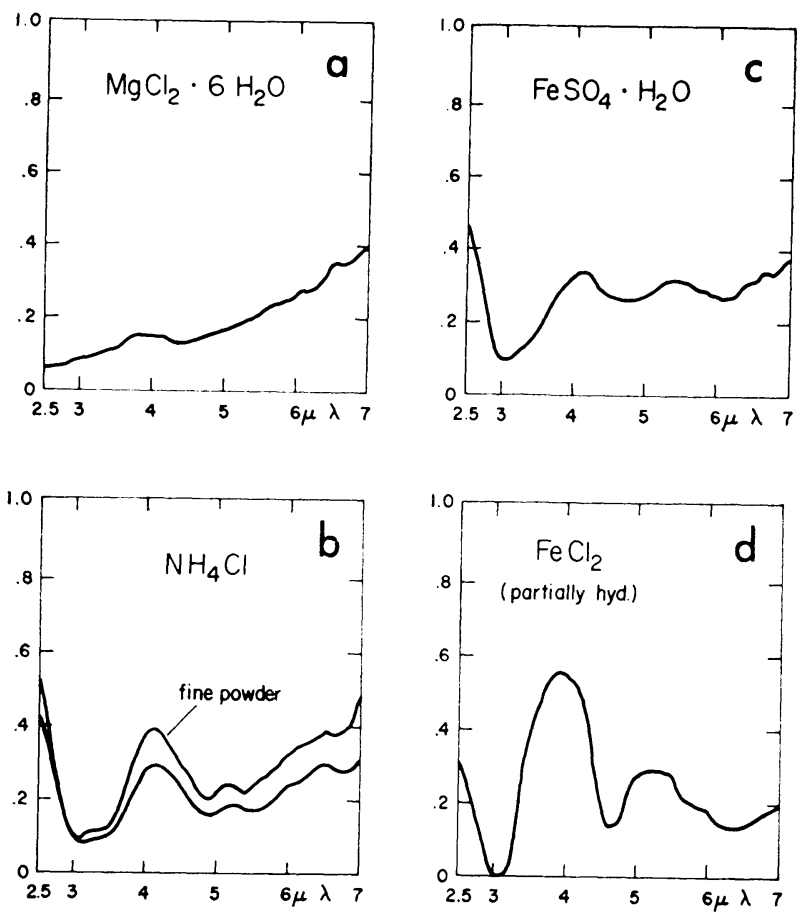

Fig. 12. Reflectance curves, $2.5-7 \mu$ for four substances.

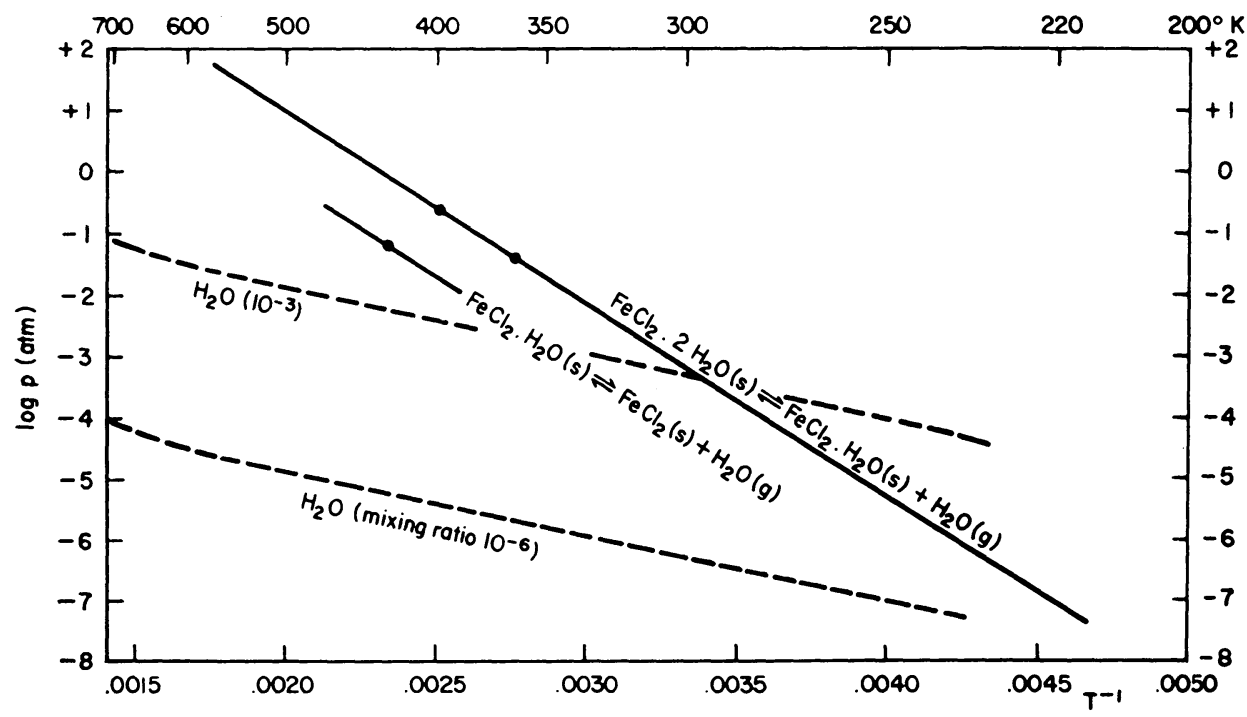

Fig. 13. Dissociation pressures for hydrated ferrous chloride in $p, T$ diagram, also showing two $\mathrm{H}_{2} \mathrm{O}$ mixing ratios for Venus atmosphere. 
about 1.55 , is in agreement with the results obtained by Coffeen for the Venus cloud particles [6].

A clinching argument favoring the identification of partially hydrated $\mathrm{FeCl}_{2}$ is found from the remarkable agreement between the dissociation pressure of $\mathrm{H}_{2} \mathrm{O}$ for the cloud material (cf. Figure 13) and the actual water-vapor pressure in the observable layers of the Venus atmosphere. If the atmosphere water-vapor content were, e.g., 100 times larger, the clouds would be tetrahydrate (Figure $11 \mathrm{c}(n)$ )) if the vapor pressure were 10 times smaller, one would expect $\mathrm{FeCl}_{2}$, anhydrous (Figure 11c $(l)$ ). Clearly, neither one satisfies the Venus reflection curve.

There are some reasons to suspect that the UV cloud layer is composed of $\mathrm{NH}_{4} \mathrm{Cl}(\mathrm{s})$ but present evidence is insufficient to establish this [4].

\section{References}

[1] CalTech-JPL Lunar and Planetary Conference, Sept. 13-18, 1965.

[2] Handbook of the Physical Properties of the Planet Venus, NASA, SP 3029, 1967.

[3] Kuiper, G. and Forbes, F.: 1967, Comm. LPL No. 95, 6, 177.

[4] Kuiper, G., Forbes, F., Steinmetz, D., and Mitchell, R.: 1968-69, Comm. LPL No. 100, 6, 209.

[5] Comm. LPL No. 95, p. 187.

[6] Coffeen, D.: 1968, Astron. J. 74, 446.

[7] Ross, F.: 1928, Astrophys. J. 67, 57.

[8] Wright, W.: 1927, Publ. Astron. Soc. Pacific 39, 220.

[9] Boyer, C. and Camichel, H.: 1967, Ann. Astrophys. 24, 531; 1965, Compt. Rend. Acad. Sci. Paris 260, 809.

[10] Boyer, C.: 1965, Astronomie 79, 223.

[11] Boyer, C. and Guérin, P.: 1966, Compt. Rend. Acad. Sci. Paris 263, 253.

[12] Kuiper, G.: 1968-69, Comm. LPL No. 101, 6, 229.

[13] Connes, et al.: 1967, Astrophys. J. 147, 1230-1237; Connes, J., Connes, P., and Maillard, J.: 1969, 'Near Infrared Spectra of Venus, Mars, Jupiter, and Saturn', CNRS, Paris.

[14] Moroz, V.: 1965, Soviet Astron.-A.J. 8, 566.

[15] de Vaucouleurs, G.: 1969, Icarus 3, 187.

\section{Discussion}

Morrison: There have been new measurements from three spacecraft of water vapor in the lower atmosphere. We suggest that some tenths of a percent water is compatible with some of the radio observations. Would you object to the presence of water clouds?

Kuiper: What I would object to is this: that one would make hypotheses believing that Venus must be a replica of the Earth. Certainly Jupiter has a very different composition from the Earth. Venus may have originated somewhat later than the Earth and therefore collected fewer volatiles. I would say that the identification of the visible clouds is definite.

However the vapor pressure of ferrous chloride at $T=240 \mathrm{~K}$ is about $10^{-23} \mathrm{~atm}$. So there is a transportation problem for the particles. And therefore, what is found in the top layer must have a bearing on what is further down. The presence of water clouds below the visible layer is very improbable.

L. Young: I want to make a comment on the $7820 \AA$ carbon dioxide band that Spinrad measured, and that you asked about. One of the reasons he thought he found a very high temperature is that he was assuming it was on the linear part of the curve of growth. Now we've made measurements from 1967 on; we have about 24 plates. And in general the temperature is about $230 \mathrm{~K}$, and we did not find any high temperatures. I believe $256 \mathrm{~K}$ was the highest temperature that we found, during that year. The phase angles varied from $26^{\circ}$ to $116^{\circ}$. I really believe that Spinrad's high temperatures are a result of the assumption about the linear part of the curve of growth. 
Kuiper: Well, I'm very happy to know that this puzzle has been solved.

Boyce: Did you in your diameter measurements take any account of the difference in size of the seeing disk between the red and blue images?

Kuiper: You see, we didn't use a disk. We used an extremely narrow crescent. I would never dare use a disk.

Suess: Did you do this experiment with a large excess of $\mathrm{CO}_{2}$ for the laboratory absorption?

Kuiper: No, we did not. 